\title{
The Action of Puricase in Depleting Stores of Serum Urate Crystals
}

\author{
B. E. A. Eno ${ }^{1}$, E. O. Effanga ${ }^{1}$, I. O. Isaac ${ }^{1} \&$ Z. Lipscey $^{1}$ \\ ${ }^{1}$ Department of Mathematics/Statistics \& Comp. Science, University of Calabar, Calabar, Cross River State, \\ Nigeria \\ Correspondence: B. E. A. Eno, Department of Mathematics/Statistics \& Comp. Science, University of Calabar, P. \\ M. B. 1115, Calabar, Cross River State, Nigeria. E-mail: enobernadette@yahoo.com
}

Received: April 15, 2012 Accepted: May 13, 2012 Online Published: July 10, 2012

doi:10.5539/jmr.v4n4p60 URL: http://dx.doi.org/10.5539/jmr.v4n4p60

\begin{abstract}
From the study of purine synthesis, urate crystal deposition in joints and puricase interaction with plasma protein, a system of first order differential equations is obtained. The existence and uniqueness of the solutions of the system of equations is established. Some controllability and stabilizability results are obtained via the Pontryagins Maximum Principles.
\end{abstract}

Keywords: Puricase, uric acid, gouty arthritis, controllability, singular perturbation

\section{Introduction}

All mammals except humans and higher apes (primates) produce the enzyme uricase (urease) which further degrades (breakdown) uric acid, leaving very low levels in the blood. All urate is digestible by uricase which converts uric acid into a more serum soluble form that can be readily excreted.

The new drug puricase contains the enzyme uricase (or urease) which rapidly eliminates stores of serum uric acid in much the same way as the hormone insulin depletes stores of glycogen in diabetes mellitus patients.

Enzymes are highly effective catalysts hence the degradation of substrates by enzymes is expected to be a fast process compared to the formation of the enzyme-substrate complex. In the steady state, the rate of formation is approximately the same as the rate of depletion of substrates (Roussel, 2005). Moreover the disappearance of urate in the blood is dependent on the quantity of urate and also on the level of puricase in the blood.

Gout is a form of arthritis (jount inflammation), that is, a disease characterized by the presence of excess (above 7 $\mathrm{mg} / \mathrm{dl}$ ) uric acid in the blood (serum). About $90 \%$ of those afflicted with gout are men over 40 years of age, while the rest are post menopausal women (Flieger, 1995). Also, of the number afflicted, at least $90 \%$ are hyperuricemic (that is, they have a serum uric acid level of above $7 \mathrm{mg} / \mathrm{dl}$ ) and $(70-75) \%$ of these cases are due to underexcretion rather than due to oversecretion (Koopman, 1993).

Uric acid, a normal by-product of purine metabolism is filtered normally by the kidneys and excreted in urine. When more uric acid is secreted than the kidneys can excrete the serum uric acid level rises. Above a concentration of $7 \mathrm{mg} / \mathrm{dl}$, the blood becomes supersaturated with needlelike crystals of a salt called monosodium urate monohydrate. These salt deposits which are the waste products from usage of protein by the body often increase in size and burst through the skin to form sinuses, discharging a chalky white material (Adelowo, 1995).

High serum uric acid concentration of more than $7 \mathrm{mg} / \mathrm{dl}$ is defined as a serum uric level of more than 2 standard deviations from the mean. This is approximately the central $95 \%$ of the standard normal distribution which lies between the limits \pm 1.96 standard deviation of its mean.

Increased synthesis of uric acid can lead to accumulation, an important cause of gout (Curto, 1998). Mbah (2001) studied glucose synthesis and insulin interaction with plasma protein in the case of diabetes mellitus. We shall carry out a similer study in the case of gouty arthritis. 


\section{Model Formulation}

Let

$x(t)$ denote the amount of uric acid in serum at time $\mathrm{t}$;

$p(t)$, the amount of puricase in serum at the same time time $t$;

$x_{0}$, the equilibrium level of uric acid in a healthy state;

$p_{0}$, the equilibrium level of uricase in a healthy state;

$g_{1}$, the rate of exogeneous supply of uric acid through food intake;

$g_{2}$, the rate of exogeneous supply of uricase, through injection;

$a_{1}$, the rate at which serum uric acid crystallizes into urate;

$a_{2}$, the rate at which the concentration of urate is decreased by uricase;

$a_{3}$, the rate at which the dosage of uricase is increased when serum uric acid level rises;

$a_{4}$, the rate at which uricase depletes stores of serum urate crystals;

$u_{f}$, uric acid from food;

$u_{p}$, uricase from puricase.

We have the following representation

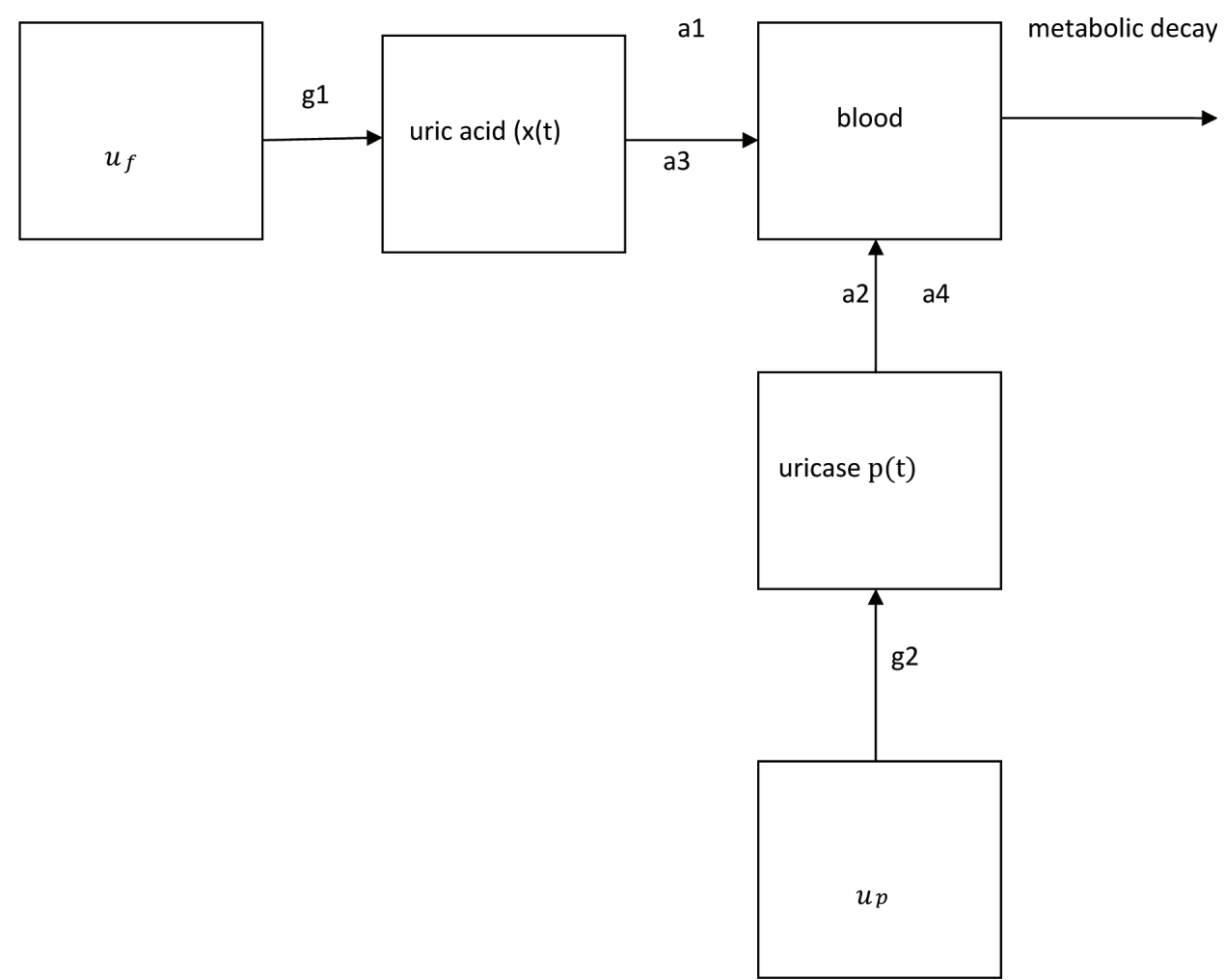

Following the information in the diagram above, the state (governing) equations have the form

$$
\left\{\begin{array}{l}
\frac{d x}{d t}=-a_{1} x-a_{2} p+g_{1} u_{f} ; x(0)=x_{0} \\
\frac{d p}{d t}=a_{3} x-a_{4} p+g_{2} u_{p} ; p(0)=p_{0} .
\end{array}\right.
$$

Hocking (1991) had earlier used the following model to describe the progression and control of diabetes mellitus:

$$
\left\{\begin{array}{l}
\frac{d g}{d t}=-c_{1} g-c_{2} h+p ; g(0)=g_{0} ; g(T)=0 \\
\frac{d h}{d t}=c_{3} g-c_{4} h+u ; h(0)=h_{0} ; h(T)=0,
\end{array}\right.
$$


where

$g(t)$ denotes the amount of glucose present at time $\mathrm{t}$;

$h(t)$, the hormone present at the same time $\mathrm{t}$;

$p(t)$, the rate of increase of glucose in the blood;

$u(t)$, the rate of increase of insulin in the blood.

System (2.1) above exhibits two mechanisms for the control of uric acid namely diet which changes the value of $g_{1}$ and medication (drug) which changes the value of $g_{2}$.

Notice that we have made use of the following facts in determining the signs of $a_{1}, a_{2}, a_{3}, a_{4}$ :

(i) When serum uric acid begins to crystallize into urate crystals which are deposited in joints and tissues, the concentration of serum uric acid decreases accordingly, hence $a_{1}>0$.

(ii) Uricase causes a decrease in the concentration of serum uric acid, hence $a_{2}>0$.

(iii) When uric acid level rises, more uricase will be injected into the blood, to increase the rate of chemical reaction, hence $a_{3}<0$.

(iv) When the enzyme/catalyst uricase increases the rate at which serum urate molecules binds with plasma protein to form serum soluble metabolites which can be promptly excreted, the concentration of uricase in the blood remains constant hence $a_{4}>0$.

\section{Model Solution}

We shall first establish the existence of bounded solutions of our system of equations by the following theorem.

Theorem 3.1 There exists bounded solutions for the system of Equation (2.1), for

$$
\left(a_{1}+a_{4}\right)^{2}<4\left(a_{4} a_{1}+a_{3} a_{2}\right) .
$$

Proof. From the first equation in system (2.1) above, we have

$$
\begin{gathered}
\frac{d x}{d t}=-a_{1} x-a_{2} p+g_{1} u_{f} \\
\Rightarrow p=\frac{1}{a_{2}}\left(-a_{1} x+g_{1} u_{f}-\frac{d x}{d t}\right) .
\end{gathered}
$$

The substitution of the value of $p$ from Equation (3.1) into the second equation in system (2.1) yields

$$
\frac{1}{a_{2}}\left(-a_{1} \frac{d x}{d t}+g_{1} \frac{d u_{f}}{d t}-\frac{d^{2} x}{d t^{2}}\right)=a_{3} x-\frac{a_{4}}{a_{2}}\left(-a_{1} x+g_{1} u_{f}-\frac{d x}{d t}\right)+g_{2} u_{p},
$$

hence

$$
a_{1} \frac{d x}{d t}+g_{1} \frac{d u_{f}}{d t}-\frac{d^{2} x}{d t^{2}}=a_{2} a_{3} x+a_{4} a_{1} x-a_{4} g_{1} u_{f}+a_{4} \frac{d x}{d t}+a_{2} g_{2} u_{p}
$$

That is

$$
\frac{d^{2} x}{d t^{2}}+\left(a_{1}+a_{4}\right) \frac{d x}{d t}+\left(a_{4} a_{1}+a_{3} a_{2}\right) x=g_{1} \frac{d u_{f}}{d t}+a_{4} g_{1} u_{f}+a_{2} g_{2} u_{p} .
$$

Let $t_{1}>0$ be the time when the exogeneous supply of serum uric acid reduces to zero. In which case $u_{f}=u_{p}=0$. Then Equation (3.2) becomes

$$
\frac{d^{2} x}{d t^{2}}+\left(a_{1}+a_{4}\right) \frac{d x}{d t}+\left(a_{4} a_{1}+a_{3} a_{2}\right) x=0
$$

The auxiliary equation for Equation (3.3) is

$$
m^{2}+\left(a_{1}+a_{4}\right) m+\left(a_{4} a_{1}+a_{3} a_{2}\right)=0
$$

For $\left(a_{1}+a_{4}\right)^{2}<4\left(a_{4} a_{1}+a_{3} a_{2}\right)$, the solution of Equation (3.3) is given by

$$
x\left(t_{1}\right)=e^{-\left(a_{1}+a_{4}\right) t}\left\{A \cos w_{0} t_{1}+B \sin w_{0} t_{1}\right\},
$$


where $w_{0}=\left\{2\left(a_{4} a_{1}+a_{3} a_{2}\right)^{\frac{1}{2}}-\left(a_{1}+a_{4}\right)\right\}$. Thus the complete solution becomes

$$
x\left(t_{1}\right)=e^{-\left(a_{1}+a_{4}\right) t}\left\{A \cos w_{0} t_{1}+B \sin w_{0} t_{1}\right\}+x_{0} .
$$

\section{Some Controllability and Stabilizability Results}

In considering the controllability and stabilizability problem of a particle whose trajectory is determined by the system

$$
\left\{\begin{array}{l}
\frac{d x}{d t}=-a_{1} x-a_{2} p+g_{1} u_{f} \\
\frac{d p}{d t}=a_{3} x-a_{4} p+g_{2} u_{p},
\end{array}\right.
$$

we shall adopt the following conditions:

(a) At time $t<t_{0}$, (where $t_{0}$ is the time when drug is first applied), we have $x<7$ which implies that no drug is applied when serum uric acid level is less than $7 \mathrm{mg} / \mathrm{dl}$.

(b) At $t=t_{0}, x\left(t_{0}\right)=x_{0}$.

(c) At final time $t_{1}$, we have $x\left(t_{1}\right)=0$ which means that drugs reduce excess serum uric acid level to zero at time $t_{1}$ implying that the patient is cured at time $t_{1}$.

We shall identify the following classes of patients:

(i) Class of patients on improper diet but with no kidney malfunction (asymptomatic stage).

(ii) Class of patients on improper diet but with kidney malfunction (critical stage).

(iii) Class of patients on proper diet and with no kidney malfunction (rest period).

(iv) Class of patients on proper diet and with kidney malfunction (hazard stage).

\section{Definition of terms}

(a) By a proper diet, we mean a combination of purine free food and drugs.

(b) By an improper diet, we mean a combination of purine rich food and drugs.

(c) By kidney malfunction, we mean the inability of the kidney to promptly excrete uric acid.

\section{Assumptions}

$A_{1}$ : Hyperuricemia is more a result of underexcretion of uric acid, than that of oversecretion of uric acid.

$A_{2}$ : All forms of underexcretion of uric acid is due to kidney malfunction.

$A_{3}$ : Drug is administered immediately after a meal, starting at $t=0$, with $p_{0}$ and $x_{0}$ as initial values of $p$ and $x$ respectively such that $p(0)=p_{0}$ and $x(0)=x_{0}$.

\section{Hypotheses}

$H_{1}$ : Patient has kidney malfunction $\Rightarrow u_{p} \neq 0$.

$\mathrm{H}_{2}$ : Patient is on proper diet $\quad \Rightarrow u_{f}=0$.

$H_{3}$ : Patient has no kidney malfunction $\Rightarrow u_{p}=0$.

$H_{4}$ : Patient is not on proper diet $\quad \Rightarrow u_{f} \neq 0$.

\section{Analysis}

Under assumption $A_{1}$, we shall consider the following cases:

Case 1 (Class of Patients on Improper Diet and with Kidney Malfunction)

In this case, $u_{f} \neq 0, u_{p} \neq 0$ and our system of equations are

$$
\left\{\begin{array}{l}
\frac{d x}{d t}=-a_{1} x-a_{2} p+g_{1} u_{f} \\
\frac{d p}{d t}=a_{3} x-a_{4} p+g_{2} u_{p},
\end{array}\right.
$$

If $u$ is the optimal combination of food and drug that can bring about a cure without fear of malnutrition or 
overdose, then we can write system (4.2) in matrix form as follows:

$$
\frac{d}{d t}\left(\begin{array}{l}
x \\
p
\end{array}\right)=\left(\begin{array}{cc}
-a_{1} & -a_{2} \\
a_{3} & -a_{4}
\end{array}\right)\left(\begin{array}{l}
x \\
p
\end{array}\right)+\left(\begin{array}{l}
g_{1} u_{f} \\
g_{2} u_{p}
\end{array}\right) .
$$

If the rate of supply $g_{1}$ of dietary purine and the rate of supply $g_{2}$ of puricase are equal to $g$ say, then our system of equations can be written as:

$$
\frac{d}{d t}\left(\begin{array}{l}
x \\
p
\end{array}\right)=\left(\begin{array}{cc}
-a_{1} & -a_{2} \\
a_{3} & -a_{4}
\end{array}\right)\left(\begin{array}{l}
x \\
p
\end{array}\right)+\left(\begin{array}{l}
u_{f} \\
u_{p}
\end{array}\right) g .
$$

Case 2 (Class of Patients on Proper Diet but with no Kidney Malfunction)

In this case, $u_{f}=0$ and $u_{p}=0$, and our system of equations become

$$
\left\{\begin{array}{l}
\frac{d x}{d t}=-a_{1} x-a_{2} p \\
\frac{d p}{d t}=a_{3} x-a_{4} p
\end{array}\right.
$$

Theorem 4.1 The zero solution of system (4.3) is a stable node if $\left(a_{1} a_{4}\right)^{2} \geq 4 a_{2} a_{3}$ and a stable focus if $\left(a_{1} a_{4}\right)^{2}<$ $4 a_{2} a_{3}$.

Proof. For the system above,

$$
\left|\begin{array}{cc}
-a_{1}-\lambda & a_{2} \\
a_{3} & -a_{4}-\lambda
\end{array}\right|=0 .
$$

On expansion, we obtain the quadratic equation

$$
\left(a_{1}+\lambda\right)\left(a_{4}+\lambda\right)+a_{2} a_{3}=0
$$

or

$$
\lambda^{2}+\left(a_{1}+a_{4}\right) \lambda+a_{1} a_{4}+a_{2} a_{3}=0 .
$$

Hence,

$$
\begin{aligned}
\lambda & =\frac{1}{2}\left\{-\left(a_{1}+a_{4}\right) \pm\left[\left(a_{1}+a_{4}\right)^{2}-4\left(a_{1} a_{4}+a_{2} a_{3}\right)\right]^{\frac{1}{2}}\right\} \\
& \left.=\frac{1}{2}\left\{-\left(a_{1}+a_{4}\right) \pm\left[\left(a_{1}-a_{4}\right)^{2}-4 a_{2} a_{3}\right)\right]^{\frac{1}{2}}\right\}
\end{aligned}
$$

Consequently, the zero solution of the linearized system is a stable node if $\left(a_{1} a_{4}\right)^{2} \geq 4 a_{2} a_{3}$ and a stable focus if $\left(a_{1} a_{4}\right)^{2}<4 a_{2} a_{3}$.

Case 3 (Class of Patients on Proper Diet but with Kidney Malfunction)

In this case, $u_{f}=0, u_{p} \neq 0$, and the only source of uric acid will be considered to stem from slight disorders in the metabolic pathway of endogeneous purine.

Our system of equations then become:

$$
\left\{\begin{array}{l}
\varepsilon \frac{d x}{d t}=-a_{1} \varepsilon x-a_{2} p \\
\frac{d p}{d t}=a_{3} \varepsilon x-a_{4} p+g_{2} u_{p},
\end{array}\right.
$$

where $\varepsilon$ represents some disorder (perturbation) in purine metabolic pathways triggered off by the use of drugs like diuretics which prevents urine retention in kidney malfunction patients. Such drugs cause dehydration and a resultant increase in the concentration of serum uric acid. To prevent such increase, more puricase has to be injected into the blood. This will in turn increase $a_{2}$. To effect a cure, $\varepsilon$ must be minimized, while $a_{2}$ must be increased by a steady increase in $u_{p}$. This may produce two effects:

(i) a blow-up (instability) of the term $\frac{a_{2}}{\varepsilon}$ with time (at infinity);

(ii) an overdose of the drug puricase.

Theorem 4.2 System (4.4) is completely controllable provided $\frac{a_{2}}{\varepsilon}$ is bounded at $\pm \infty$ and the optimal dosage of puricase required to prevent an overdose is $-\frac{p_{2} g_{2}}{2 e^{a_{1}}}$. 
Proof. We rewrite system (4.4) in matrix form as

$$
\frac{d}{d t}\left(\begin{array}{l}
x \\
p
\end{array}\right)=\left(\begin{array}{cc}
-a_{1} & \frac{-a_{2}}{\varepsilon} \\
a_{3} \varepsilon & -a_{4}
\end{array}\right)\left(\begin{array}{l}
x \\
p
\end{array}\right)+\left(\begin{array}{c}
0 \\
g_{2}
\end{array}\right) u_{p}
$$

or

$$
\frac{d X}{d t}=A X+B u_{p}
$$

This implies that

$$
B u_{p} d t=d X-A X d t
$$

where

$$
X=\left(\begin{array}{l}
x \\
p
\end{array}\right) ; \quad A=\left(\begin{array}{cc}
-a_{1} & \frac{-a_{2}}{\varepsilon} \\
a_{3} \varepsilon & -a_{4}
\end{array}\right) \text { and } B=\left(\begin{array}{c}
0 \\
g_{2}
\end{array}\right) .
$$

The controllability matrix for system (4.5) is given by

$$
C=\left[\begin{array}{lllll}
B & A B & A^{2} B & \cdots & A^{n-1} B
\end{array}\right]=\left(\begin{array}{cc}
0 & \frac{-a_{2} g_{2}}{\varepsilon} \\
g_{2} & -a_{4} g_{2}
\end{array}\right) .
$$

The matrix $\mathrm{C}$ has two linearly independent rows (that is $\mathrm{C}$ is of rank 2), and $\mathrm{A}$ has order 2. Therefore we arrive at the following conclusions:

(i) That system (4.5) is completely controllable implying that complications such as chronic topheceous gouty arthritis which may lead to stiffness, crippling and deformity can be prevented and hence cure may be effected in finite time through drug action, for a patient on proper diet.

(ii) Subsequent attacks following an initial episode can be arrested in finite time. From the equation

$$
\varepsilon \frac{d x}{d t}=-a_{1} \varepsilon x-a_{2} p
$$

we have

$$
\varepsilon \frac{d x}{d t}+-a_{1} \varepsilon x=-a_{2} p
$$

which implies that

$$
x=e^{-a_{1} t} \int_{0}^{t}\left(\frac{-a_{2} p}{\varepsilon}\right) e^{a_{1} \tau} d \tau .
$$

thus, safe levels of uric acid may be attained by using large doses of drugs, but this may induce dangerous sideeffects. Moreover, such drugs may be rather too expensive.

To balance up the two effects, Hocking (1991) employed the following cost functional

$$
J=\frac{1}{2} \int_{0}^{t_{1}}\left(g^{2}+k^{2} u^{2}\right) d t,
$$

where $t_{1}$ is the equilibrium time during which $\mathrm{u}$ is reduced to zero, $g$ is the amount of glucose, $u$ is the amount of insulin and $k^{2}$ is the weighting factor between the two cost components. Employing Equation (4.6) above, and modeling after Hocking (1991), we shall define our cost function as

$$
J=\int_{0}^{t_{1}}\left(k^{2} u_{f}^{2}+u_{p}^{2}\right) e^{a_{1} t} d t,
$$

where $u_{f}$ and $u_{p}$ are defined as in section 2 and $a_{1}$ represents the rate at which serum uric acid crystallizes into urate.

Exponential decay stems from the fact that the concentration of serum uric acid decreases in a simple exponential way, as uric acid crystallizes into urate salt, which settles in the joints and tissues with $k$ as the relative importance of malnutrition and overdose. 
We shall consider the fixed endpoint problem with sufficiently large upper bound on $g_{2}$ and with $x\left(t_{1}\right)$ and $p\left(t_{1}\right)$ fixed, in which case $x\left(t_{1}\right)$ is just a point on $R^{n}$ and $p\left(t_{1}\right)$ is another point on the same $R^{n}$.

In the fixed endpoint problem, terminal conditions are required. Hence we shall introduce the adjoint (co-state) variables $p_{1}$ and $p_{2}$ and state our terminal conditions as follows

$$
p_{1}\left(t_{1}\right)=p_{2}\left(t_{2}\right)=0 .
$$

Our next task is to minimize the cost function. This is achieved by first obtaining the optimal control function and using same to replace all the control variables in the subsequent equations.

It is worthy of note that the carrier of the optimal control function in question is the Hamiltonian which in the study by O'malley and Kung (1975) is represented by the equation

$$
\begin{aligned}
H\left(x, p, p_{1}, p_{2}, u, t\right) & =\frac{\partial J}{\partial t}+\langle\text { co }- \text { state variable, system equations }\rangle \\
& =\frac{\partial J}{\partial t}+\left\langle\left(p_{1}, p_{2}\right),(A, B)\right\rangle,
\end{aligned}
$$

where the angled brackets $\langle\cdot, \cdot\rangle$ represent a scalar product. In the context under consideration, the Hamiltonian takes the form

$$
H=\left(k^{2} u_{f}^{2}+u_{p}^{2}\right) e^{a_{1} t}+p_{1}\left(-a_{1} \varepsilon x-a_{2} p\right)+p_{2}\left(a_{3} \varepsilon x-a_{4} p+g_{2} u_{p}\right) .
$$

Now

$$
\varepsilon \frac{\partial p_{1}}{\partial t}=-\frac{\partial H}{\partial x}=p_{1} a_{1} \varepsilon-p_{2} a_{3} \varepsilon
$$

and

$$
\varepsilon \frac{\partial p_{2}}{\partial t}=-\frac{\partial H}{\partial p}=p_{1} a_{2}+p_{2} a_{4}
$$

Along the optimal trajectory, the derivative of the Hamiltonian with respect to the control variable $u_{p}$ must be equals to zero, that is,

$$
\frac{\partial H}{\partial u_{p}}=0,
$$

a condition typified by PONTRYAGIN'S MAXIMUM PRINCIPLE. By virtue of this principle, Equation (4.8) is quickly reduced to the form

$$
2 e^{a_{1} t} u_{p}+p_{2} g_{2}=0
$$

hence,

$$
u_{p}=-\frac{p_{2} g_{2}}{2 e^{a_{1} t}} .
$$

Furthermore, since

$$
\frac{\partial^{2} H}{\partial u_{p}^{2}}=2 e^{-t}>0
$$

then our $u_{p}$ will minimize $\mathrm{J}$. Therefore $u_{p}$ is an OPTIMAL CONTROL, since it minimizes $\mathrm{J}$ over all admissible inputs (controls).

\section{Case 4 (The Critical Case)}

In this case, two conditions abound:

(a) the rate $a_{1}$ at which serum uric acid crystallizes into urate crystals is so high that $a_{1} \varepsilon$ does not tend to zero as $\varepsilon \rightarrow 0$.

(b) the corresponding rate $a_{3}$ at which the concentration of the drug puricase is increasing is so high that $a_{3} \varepsilon$ does not tend to zero as $\varepsilon \rightarrow 0$.

We shall let $a_{1} \varepsilon=\alpha$ and $a_{3} \varepsilon=\beta$ so that system (4.4) now becomes

$$
\left\{\begin{array}{l}
\varepsilon \frac{d x}{d t}=-\alpha x-a_{2} p \\
\frac{d p}{d t}=\beta x-a_{4} p+g_{2} u_{p}
\end{array}\right.
$$


and Equations (4.7) and (4.8) can be written as

$$
\left\{\begin{array}{l}
\varepsilon \frac{d p_{1}}{d t}=\alpha p_{1}-\beta p_{2} \\
\frac{d p_{2}}{d t}=a_{2} p_{1}+a_{4} p_{2} .
\end{array}\right.
$$

Substituting $u_{p}=-\frac{1}{2} e^{-a_{1} t} g_{2} p_{2}$ into system (4.9), we obtain the following system of equations

$$
\left\{\begin{array}{l}
\varepsilon \frac{d x}{d t}=-\alpha x-a_{2} p \\
\frac{d p}{d t}=\beta x-a_{4} p-\frac{1}{2} g_{2}^{2} e^{-a_{1} t} p_{2} .
\end{array}\right.
$$

In matrix form we have

$$
\frac{d}{d t}\left(\begin{array}{c}
x \\
p \\
p_{1} \\
p_{2}
\end{array}\right)=\left(\begin{array}{cccc}
-a_{1} & -\frac{a_{2}}{\varepsilon} & 0 & 0 \\
a_{3} \varepsilon & -a_{4} & 0 & -\frac{1}{2}\left(g e^{-a_{1} t} p_{2}\right) \\
0 & 0 & a_{1} & -a_{3} \\
0 & 0 & a_{2} & a_{4}
\end{array}\right)\left(\begin{array}{c}
x \\
p \\
p_{1} \\
p_{2}
\end{array}\right)
$$

In the limit as $\varepsilon \rightarrow 0$, we have the reduced system

$$
\left\{\begin{array}{l}
0=-\alpha x-a_{2} p \\
\frac{d p}{d t}=\beta x-a_{4} p-\frac{1}{2} g_{2}^{2} e^{-a_{1} t} p_{2} \\
0=\alpha p_{1}-\beta p_{2} \\
\frac{d p_{2}}{d t}=a_{2} p_{1}+a_{4} p_{2} .
\end{array}\right.
$$

Let $\left(x_{0}, p_{0}, p_{10}, p_{20}\right)$ be the solution of the reduced (limiting) problem $(\varepsilon \rightarrow 0)$, then the subsystem,

$$
\left\{\begin{array}{l}
0=-\alpha x_{0}-a_{2} p_{0} \\
0=\alpha p_{10}-\beta p_{20}
\end{array}\right.
$$

of the reduced problem can be written in matrix form as

$$
\left(\begin{array}{cc}
a_{2} & 0 \\
0 & \beta
\end{array}\right)\left(\begin{array}{c}
p_{0} \\
p_{20}
\end{array}\right)=\left(\begin{array}{cc}
-\alpha & 0 \\
0 & a_{1}
\end{array}\right)\left(\begin{array}{c}
x_{0} \\
p_{10}
\end{array}\right) .
$$

The coefficient matrices in (4.15) are nonsingular in the sense that the following conditions are satisfied:

$$
\left|\begin{array}{cc}
a_{2} & 0 \\
0 & \beta
\end{array}\right| \neq 0 \text { and }\left|\begin{array}{cc}
-\alpha & 0 \\
0 & a_{1}
\end{array}\right| \neq 0 \text {. }
$$

Therefore the reduced system has a unique solution.

Case 5 (Puricase is Perturbed by the Action of Certain Inhibiting Enzymes)

In this case, our system of equations become

$$
\left\{\begin{array}{l}
\frac{d x}{d t}=-\alpha x-\varepsilon a_{2} p \\
\varepsilon \frac{d p}{d t}=a_{3} x-\varepsilon a_{4} p-\frac{1}{2} g_{2}^{2} e^{-a_{1} t} p_{2} \\
\frac{d p_{1}}{d t}=\alpha p_{1}-\beta p_{2} \\
\varepsilon \frac{d p_{2}}{d t}=\varepsilon a_{2} p_{1}+\varepsilon a_{4} p_{2} .
\end{array}\right.
$$

The reduced problem is

$$
\left\{\begin{array}{l}
0=a_{3} x-\varepsilon a_{4} p-\frac{1}{2} g_{2}^{2} e^{-a_{1} t} p_{2} \\
0=\varepsilon a_{2} p_{1}+\varepsilon a_{4} p_{2} .
\end{array}\right.
$$

From the first equation in system (4.17), we have

$$
a_{3} x=\varepsilon a_{4} p+\frac{1}{2} g_{2}^{2} e^{-a_{1} t} p_{2}
$$

or

$$
a_{3} x=\varepsilon a_{4} p-g_{2} u_{p}
$$


From Equation (4.18), we shall conclude that at the point when all inhibiting factors have been eliminated $(\varepsilon=0)$, continuous application of puricase (in which case $a_{4}=0$ ), will progressively (in the limit as $t \rightarrow \infty$ ) eliminate stores of serum uric acid.

\section{Case 6 (The Hazardous Case)}

This case may stem from genetic or hereditary factors or from other disease conditions. In this case serum uric acid level becomes very high, and we may adopt a cost functional that is linear in $u_{p}$, and quadratic in $x$. We shall define our cost functional as

$$
J=\int_{0}^{t_{1}} e^{-a_{1} t}\left(k_{1} x^{2}+k_{2} u_{p}\right) d t
$$

and our optimal control problem shall be defined as:

$$
\text { Minimize } u \in\left[u_{1}, u_{2}\right] \int_{0}^{t_{1}} e^{-a_{1} t}\left(k_{1} x^{2}+k_{2} u_{p}\right) d t
$$

subject to system (4.4), where $k_{1}$ is the cost of prevention and $k_{2}$ is the cost of treatment.

Since the cost $k_{2}$ of treatment is directly proportional to the rate $g_{2}$ of supply of the drug puricase, we shall let $k_{2}=\alpha g_{2}$, where $\alpha$ is an arbitrary constant. Moreover, the aim of treatment is to prevent an attack of gout and the $\operatorname{cost} k_{1}$ of prevention is directly proportional to the cost $k_{2}$ of treatment. That is, $k_{1}=\beta \alpha g_{2}$.

Theorem 4.3 The optimal amount of serum uric acid resulting from proper diet and optimal dosage of puricase depends on the rate $a_{1}$ at which serum uric acid crystallizes into urate.

Proof. Employing the facts above, we can rewrite our cost functional as

$$
J=\alpha g_{2} \int_{0}^{t_{1}} e^{-a_{1} t}\left(k_{1} x^{2}+u_{p}\right) d t
$$

Corresponding to system (4.4), the Hamiltonian function to be minimized is given by:

$$
H\left(x, p, p_{1}, p_{2}, u_{p}, t\right)=e^{-a_{1} t}\left(k_{1} x^{2}+k_{2} u_{p}\right)+p_{1}\left(-a_{1} x-\frac{a_{2} p}{\varepsilon}\right)+p_{2}\left(-a_{4} p+g_{2} u_{p}\right)
$$

Differentiating the first equation in system (4.4) once with respect to t yields:

$$
\varepsilon \frac{d^{2} x}{d t^{2}}=-a_{1} \varepsilon \frac{d x}{d t}-a_{2} \frac{d p}{d t} .
$$

Substituting the value of $\frac{d p}{d t}$ from the second equation of system (4.4) into Equation (4.19) yields:

$$
\varepsilon \frac{d^{2} x}{d t^{2}}=-a_{1} \varepsilon \frac{d x}{d t}-a_{2}\left(a_{3} \varepsilon x-a_{4} p+g_{2} u_{p}\right)
$$

which implies

$$
-\varepsilon \frac{d^{2} x}{d t^{2}}=a_{1} \varepsilon d x+a_{2}\left(a_{3} \varepsilon x-a_{4} p+g_{2} u_{p}\right) d t
$$

or

$$
a_{2}\left(a_{3} \varepsilon x-a_{4} p+g_{2} u_{p}\right) d t=-\varepsilon \frac{d^{2} x}{d t^{2}}=a_{1} \varepsilon d x
$$

Hence,

$$
u_{p} d t=\frac{1}{g_{2}}\left\{-\varepsilon \frac{d^{2} x}{d t^{2}} d t-a_{1} \varepsilon d x-a_{2}\left(a_{3} \varepsilon x-a_{4} p\right) d t\right\} .
$$

Substituting the value of $u_{p} d t$ derived from Equation (4.20) into our cost functional, we obtain the line integral

$$
J_{C}=\alpha g_{2} \int_{C} e^{-a_{!} t}\left[k_{1} x^{2} d t+\frac{1}{g_{2}}\left\{-\varepsilon \frac{d^{2} x}{d t^{2}} d t-a_{1} \varepsilon d x-a_{2}\left(a_{3} \varepsilon x-a_{4} p\right) d t\right\}\right]
$$


along any curve $\mathrm{C}$ in the $(x, t)$ space. We shall apply the Green's theorem to our line integral over an area $R$ bounded by $C$ to obtain:

$$
J_{C}=\alpha g_{2} \iint_{R}\left[-\frac{\partial}{\partial t}\left(a_{1} \varepsilon e^{-a_{1} t}\right)+\frac{\partial}{\partial x} e^{-a_{1} t}\left\{k_{1} x^{2} d t-\frac{\varepsilon}{g_{2}} \frac{d^{2} x}{d t^{2}}-a_{2} a_{3} \varepsilon x+a_{2} a_{4} p\right\}\right] d t d x
$$

or

$$
J_{C}=\alpha g_{2} \iint_{R}\left[-\frac{\partial}{\partial t}\left(\varepsilon_{1} e^{-a_{1} t}\right)+\frac{\partial}{\partial x}\left(e^{-a_{!} t}\right)\left(e^{-a_{!} t}\right) *\left\{k_{1} x^{2} d t-\frac{\varepsilon}{g_{2}} \frac{d^{2} x}{d t^{2}}-a_{2} a_{3} \varepsilon x+a_{2} a_{4} p\right\}\right] d t d x
$$

where $\varepsilon_{1}=a_{1} \varepsilon$.

As it stands, $\varepsilon \rightarrow 0$ faster than $\varepsilon_{1}$. Hence in the limit as $\varepsilon \rightarrow 0$, Equation (4.21) becomes

$$
\begin{aligned}
J_{C} & =\alpha g_{2} \iint_{R}\left[-\frac{\partial}{\partial t}\left(\varepsilon_{1} e^{-a_{1} t}\right)+\frac{\partial}{\partial x}\left(e^{-a_{1} t}\right)\left\{k_{1} x^{2}+a_{2} a_{4} p\right\}\right] d t d x \\
& =\iint_{R} \alpha g_{2}\left[a_{1} \varepsilon_{1} e^{-a_{1} t}+\left(e^{-a_{t} t}\right)\left(2 k_{1} x\right)\right] d t d x
\end{aligned}
$$

To specify our optimal control, we need to partition the $\left(t, x_{1}\right)$ space into regions where the integrand in Equation (4.22) above takes positive and negative values. Equating the integrand in Equation (4.22) to zero, we have:

$$
\begin{aligned}
\alpha g_{2}\left[a_{1} \varepsilon_{1} e^{-a_{1} t}+\left(e^{-a_{1} t}\right)\right. & \left.\left(2 k_{1} x\right)\right]=0 \\
\Rightarrow 2 k_{1} x & =-a_{1} \varepsilon_{1} \\
\Rightarrow x_{\text {opt }} & =-\frac{a_{1} \varepsilon_{1}}{2 k_{1}}
\end{aligned}
$$

\section{Application of Boundary Layer Analysis to a Singularly Perturbed Model of the Gout Disease}

Gout is a metabolic disorder in which excess uric acid is produced and deposited mostly in the joints. Note that a joint depicts the junction or boundary between two bones.

In this section we shall carry out some boundary layer analysis on our singularly perturbed model of the gout disease. Generally substrate (urate) velocity increases across the boundary layer (the joint), causing a corresponding increase in temperature around the affected joint, which in turn may lead to an attack. This increase in temperature in turn increases the particle deposition rate (Joshua, 2008).

Fluid velocity increases across the boundary layer, reaching its peak value and then decreasing to the free stream velocity value. Possible heat generation effects may alter the temperature distribution and the particle deposition rate.

Boundary layer analysis involves the matching of the inner (fast transient) solution $x_{i}(\tau, \varepsilon), y_{i}(\tau, \varepsilon)$ (also called the boundary layer correction), which approaches zero and the outer (slow decay) solution $x_{0}(t, \varepsilon), y_{0}(t, \varepsilon)$. The matching is done through a stretching (straining) transformation $\tau=\frac{t}{\varepsilon}$. Since they just represent two pieces of the same trajectory, the inner and outer solutions have to match up in an intermediate time range, through the stretching (straining) transformation. In composite form

$$
\left(\begin{array}{l}
x \\
y
\end{array}\right)=\left(\begin{array}{l}
X(t, \varepsilon) \\
Y(t, \epsilon)
\end{array}\right)+\left(\begin{array}{l}
\xi(\tau, \varepsilon) \\
\eta(\tau, \varepsilon)
\end{array}\right)
$$

with the outer expansion

$$
\left(\begin{array}{c}
X(t, \varepsilon) \\
Y(t, \epsilon)
\end{array}\right) \sim \sum_{j=0}^{\infty}\left(\begin{array}{c}
X_{j}(t) \\
Y_{j}(t)
\end{array}\right) \varepsilon^{j}
$$

and the initial (boundary) layer correction

$$
\left(\begin{array}{l}
\xi(\tau, \varepsilon) \\
\eta(\tau, \epsilon)
\end{array}\right) \sim \sum_{j=0}^{\infty}\left(\begin{array}{l}
\xi_{j}(\tau) \\
\eta_{j}(\tau)
\end{array}\right) \varepsilon^{j}
$$


whose terms all decay to zero as the stretched variable $\tau=\frac{t}{\varepsilon}$ tends to $\infty$. Then the asymptotic solution for each fixed $t>0$ will be given to all orders by the outer solution. The outer solution is supposed to be valid when is not too small while the inner solution is valid when $\tau$ is not too large (Roussel, 2005).

In our analysis, the function $\left\{x_{i}\left(\frac{t}{\varepsilon}, \varepsilon\right), y_{i}\left(\frac{t}{\varepsilon}, \varepsilon\right)\right\}$ will be called the boundary layer solution (or correction term). Our controllable process shall be partitioned into 2 phases namely: The build up of high serum urate levels (triggers), and the onset of gouty arthritis ( $\left(1^{s t}\right.$ attack). Whereas the build up is slow, the onset of attack is fast and can only be described in a continuous time model. We shall therefore identify two adjoining regions (subdomains) and two time variables namely: a slow time variable $(t)$ and a fast time variable $(\tau)$.We shall consider two adjoining domains: an inner (boundary layer, $0<t<t_{0}$ ) domain representing the ligament and an outer $\left(t_{0}<t<t_{1}\right)$ domain representing the tendon.

From equation (4.20), we have

$$
\varepsilon \frac{d^{2} x}{d t^{2}} d t=-a_{1} \varepsilon d x-a_{2}\left(a_{3} \varepsilon x-a_{4} p+g_{2} u_{p}\right) d t .
$$

We shall assume that $a_{4} \rightarrow 0$ as $\rightarrow t_{1}$, in which case a single dose therapy can be employed towards the end of the treatment. In this case, Equation (5.1) becomes:

$$
\varepsilon \frac{d^{2} x}{d t^{2}}=-a_{1} \varepsilon \frac{d x}{d t}-a_{2}\left(a_{3} \varepsilon x+g_{2} u_{p}\right)
$$

or

$$
\varepsilon \frac{d^{2} x}{d t^{2}}=-\alpha \frac{d x}{d t}-a_{2}\left(\beta x+g_{2} u_{p}\right) .
$$

In the outer domain, the leading term $\varepsilon \frac{d^{2} x}{d t^{2}}$ becomes more and more negligible, as $\varepsilon$ becomes smaller and smaller $(\varepsilon \rightarrow 0)$. Hence, provided $x$ is finitely differentiable (that is provided the disease is controllable), we shall consider the equation

$$
-\alpha \frac{d x^{0}}{d t}-a_{2}\left(\beta x^{0}+g_{2} u_{p}\right)=0
$$

which implies

$$
u_{p}=\frac{1}{a_{2} g_{2}}\left(a_{2} \beta x^{0}+\alpha \frac{d x^{0}}{d t}\right)
$$

in the outer domain. Here the superscript ${ }^{0}$ denotes the outer region and the inner boundary condition $x_{1}\left(t_{0}\right)=x_{10}$ has been dropped.

In the inner (boundary layer) domain however, where changes are rapid, $\frac{d^{2} x}{d t^{2}}$ becomes so large that the $\varepsilon \frac{d^{2} x}{d t^{2}}$ term cannot be neglected as $\varepsilon \rightarrow 0$ and so we consider the equation

$$
\varepsilon \frac{d^{2} x^{i}}{d t^{2}}=-\alpha \frac{d x^{i}}{d t}-a_{2}\left(\beta x^{i}+g_{2} u_{p}\right)
$$

or

$$
u_{p}=-\frac{1}{a_{2} g_{2}}\left(\varepsilon \frac{d^{2} x^{i}}{d t^{2}}+\alpha \frac{d x^{i}}{d t}+a_{2} \beta x^{i}\right)
$$

To develop a mathematical model that can slow down fast transients in the inner, narrow boundary layer region of the singularly perturbed model of the disease gout (a phenomenon which depicts a reduction of the amount of uric acid in the blood and a consequent reduction of the effect of the gout disease), we shall let

$$
x^{i}(t, \varepsilon)=x_{10}^{i}(t)+\varepsilon x_{11}^{i}(t)+\varepsilon^{2} x_{12}^{i}(t)+\cdots
$$

and

$$
u(t, \varepsilon)=u_{1}(t)+\varepsilon u_{1}(t)+\varepsilon^{2} u_{3}(t)+\cdots
$$

Equations (5.4) and (5.5) are nave perturbation expansions. The superscript ${ }^{i}$ denotes the inner region, and the outer boundary condition $x_{1}^{0}\left(t_{1}\right)=0$ has been dropped.

We shall introduce a stretching transformation $\tau=\frac{t}{\varepsilon^{\beta}}$, where $\beta>0$. This transformation magnifies the time variable $t$ as $\varepsilon \rightarrow 0$ in the sense that $t \rightarrow \infty$ as $\varepsilon \rightarrow 0$. Finally, we shall choose $\beta$ to be sufficiently large such 
that $\left(x_{1}^{i}\right)^{\prime}=\frac{d x_{1}^{i}}{d \tau}$ and $\left(x_{1}^{i}\right)^{\prime \prime}=\frac{d^{2} x_{1}^{i}}{d \tau^{2}}$ are bounded. In this way the various terms in our asymptotic expansions can be ordered in magnitude by comparing the powers of $\varepsilon$ by which they were multiplied.

Now

$$
\frac{d x^{i}}{d t}=\frac{d x^{i}}{d \tau} \cdot \frac{d \tau}{d t}=\varepsilon^{-\beta}\left(x_{1}^{i}\right)^{\prime}
$$

and

$$
\begin{aligned}
\frac{d^{2} x^{i}}{d t^{2}} & =\frac{d}{d \tau} \cdot \frac{d \tau}{d t}\left[\varepsilon^{-\beta} \frac{d x^{i}}{d \tau}\right] \\
& =\varepsilon^{-\beta}\left[\varepsilon^{-\beta} \frac{d^{2} x^{i}}{d \tau^{2}}\right]=\varepsilon^{-2 \beta}\left(x_{1}^{i}\right)^{\prime \prime}
\end{aligned}
$$

Substituting the expansions obtained above into Equation (5.3), we arrive at the following equation:

$$
u_{p}=\frac{1}{a_{2} g_{2}}\left(-\varepsilon\left(x_{1}^{i}\right)^{\prime \prime}-a_{1}\left(x_{1}^{i}\right)^{\prime}-a_{2} a_{3}\left(x_{1}^{i}\right)\right)
$$

Theorem 5.1 The control $B^{T}(\tau) \phi^{T}\left(t_{0}, \tau\right) x_{0} \phi^{\prime}\left(t_{0}, \tau\right)$ leaves the system

$$
\left\{\begin{array}{l}
\frac{d x}{d t}=A(t) x(t)+B(t) u(t) \\
x\left(t_{0}\right)=x_{0}
\end{array}\right.
$$

invariant.

Proof.

$$
\left\{\begin{array}{l}
\dot{x}=A(t) x(t)+B(t) u(t) \\
x\left(t_{0}\right)=x_{0}
\end{array}\right.
$$

this means that

$$
x(t)=\phi\left(t, t_{0}\right)\left[x_{0}+\int_{t_{0}}^{t} \phi\left(t_{0}, \tau\right) B(\tau) u(\tau) d \tau\right],
$$

where

$$
\phi\left(t, t_{0}\right)=e^{\left(\int_{t_{0}}^{t} A(t) d t\right)}
$$

is the state transition matrix. At $t=t_{1}$, we have

$$
x\left(t_{1}\right)=\phi\left(t_{1}, t_{0}\right)\left[x_{0}+\int_{t_{0}}^{t_{1}} \phi\left(t_{0}, \tau\right) B(\tau) u(\tau) d \tau\right] .
$$

Replacing $u(t)$ with $B^{T}(\tau) \phi^{T}\left(t_{0}, \tau\right) x_{0} \phi^{\prime}\left(t_{0}, \tau\right)$ in Equation (5.7) above, we obtain

$$
\begin{aligned}
x\left(t_{1}\right) & =\phi\left(t_{1}, t_{0}\right)\left[x_{0}+\int_{t_{0}}^{t_{1}} \phi\left(t_{0}, \tau\right) B(\tau) B^{T}(\tau) \phi^{T}\left(t_{0}, \tau\right) x_{0} \phi^{\prime}\left(t_{0}, \tau\right) d \tau\right] . \\
& =\phi\left(t_{1}, t_{0}\right)\left[x_{0}+x_{0} \int_{t_{0}}^{t_{1}} \phi^{\prime}\left(t_{0}, \tau\right) d \tau\right] \\
& =\phi\left(t_{1}, t_{0}\right)\left[x_{0}+x_{0}\left\{\phi\left(t_{0}, t_{1}\right)-\left(t_{0}, t_{0}\right)\right\}\right] \\
& =\phi\left(t_{1}, t_{0}\right)\left[x_{0}+x_{0} \phi\left(t_{1}, t_{0}\right)-x_{0}\right] \\
& =\phi\left(t_{1}, t_{0}\right) \cdot x_{0} \phi\left(t_{0}, t_{1}\right) \\
& =\phi\left(t_{1}, t_{1}\right) \cdot x_{0}=x_{0} \\
& \equiv x\left(t_{0}\right) .
\end{aligned}
$$


To slow down the fast transients $\left(x^{i}\right)^{\prime}$ and $\left(x^{i}\right)^{\prime \prime}$ in Equation (5.6), we introduce a new control $u\left(t-t^{*}, \varepsilon\right)=$ $B^{T}\left(t-t^{*}\right) \phi^{T}\left(t_{0}, t-t^{*}\right) x_{0} \phi^{\prime}\left(t_{0}, t-t^{*}\right)$ in Theorem 5.1 above and consiquently transform Equation (5.6) into the following statement

$$
-\varepsilon^{1-2 \beta}\left(x^{i}\right)^{\prime \prime}-a_{1} \varepsilon^{-\beta}\left(x^{i}\right)^{\prime}-a_{2} a_{3}\left(x^{i}\right)=B^{T}\left(t-t^{*}\right) \phi^{T}\left(t_{0}, t-t^{*}\right) x_{0} \phi^{\prime}\left(t_{0}, t-t^{*}\right)
$$

where $\phi\left(t, t_{0}\right)=\exp \left(\int_{t_{0}}^{t} A(t) d t\right)$ is the state transition matrix, ()$\left.^{\prime}\right)=\frac{d}{d t},\left(^{\prime}\right)=\frac{d}{d \tau},\left({ }^{T}\right)$ denotes the transposition matrix, $\left(t-t^{*}\right)<<\varepsilon$ denotes the time lag, $u\left(t-t^{*}\right)=B^{T}\left(t-t^{*}\right) \phi^{T}\left(t-t^{*}, t_{0}\right) x_{0} \phi^{\prime}\left(t-t^{*}, t_{0}\right)$ is the slow control and $\beta$ strikes the balance between food and drug input (rate of exogeneous supply of urate) and the rate of recovery (rate of elimination of urate).

In order to attain this balance, we shall equate the corresponding powers of $\varepsilon$ on both sides of Equation (5.7), to obtain $1-2 \beta=-\beta$ or $\beta=1$ such that Equation (5.7) now becomes

$$
-\varepsilon^{-1}\left(x^{i}\right)^{\prime \prime}-a_{1} \varepsilon^{-1}\left(x^{i}\right)^{\prime}-a_{2} a_{3}\left(x^{i}\right)=B^{T}\left(t-t^{*}\right) \phi^{T}\left(t-t^{*}, t_{0}\right) x_{0} \dot{\phi}\left(t-t^{*}, t_{0}\right)
$$

\section{Summary and Conclusion}

That the Hamiltonian (in Equation 4.18) is linear in $u$ implies that our optimal control $u^{*}(t)$ is bang-bang (Sethi \& Staats, 1978). That is

$$
u^{*}(t)= \begin{cases}0 & \text { if } x<7 \\ u_{1} & \text { if } x=7 \\ u_{2} & \text { if } x>7\end{cases}
$$

This implies that at the hazardous stage, when puricase is not applied continuously, the health status of the patient fluctuates back and forth between a steady state health level and an unhealthy one.

(i) From Equation (4.18), we conclude that at the point when all inhibiting factors must have been eliminated $(\varepsilon=0)$, continuous application of puricase (in which case $a_{4}=0$ ) will progressively (in the limit as $t \rightarrow t_{1}$ ) deplete stores of serum uric acid. A single dose therapy of puricase may therefore be applied continuously until stores of urate crystals are completely eliminated.

(ii) Subsequent attacks of gout following an initial episode can be arrested in finite time and complications such as Chronic Topheceous Gouty Arthritis which may lead to stiffness, crippling and deformity can be prevented and cure can be effected in finite time through the action of the drug puricase, for a patient on proper diet.

\section{References}

Adelowo, Femi. (1995). Arthritis \& Rheumatism. A Christy David Group Publication.

Barnett, S. (1975). Introduction to Mathematical Control Theory. Oxford: Clarendon Press.

Curto. (1998). Biochemistry Journal, 329, 477 - 487.

Eke, A. N. (2000). Stabilizability for Linear Feedback Observable Systems. Journal of the Nigerian Mathematical Society, 19, $59-68$.

Flieger, K. (1995). Getting to know Gout, FDA Consumer, BNET.com Gout, from Wikipedia, the free encyclopedia.

Hocking, L. M. (1991). An Introduction to the Theory of Optimal Control with Applications. Oxford: Clarendon Press.

Jacobs, O. L. R. (1974). Introduction to Control Theory. Oxford: Clarendon Press.

Joshua, E. E. (2008). Drug Absorption in the Gastrointestinal Tract: A Mathematical Model. Journal of the Nigerian Mathematical Society, 27, 109 - 122.

Kimbir, A. R., Chiawa, M., \& Kembe, M. (2003). A Mathematical Model for a Stable Students Population Dynamics in Nigerian Universities. Abacus: The Journal of the Mathematical Association of Nigeria, 30(2A), $88-93$.

Koopman, W. J. (1993). Arthritis and Allied Conditions. A textbook of rheumatology (13th ed.). Vol. 1, 1 - 1305. 
Liadi, M. A. (1999). A Qualitative Study of Andrew Dobsons Disease. A Ph. D dissertation (unpublished).

Mbah, G. C. E. (2001). An Analytical Method of Solution to the Generalized Mathematical Model used for the Study of Insulin Dependent Diabetes Mellitus. Journal of the Nigerian Mathematical Society, 20, 65 - 76.

O’malley, R. E. O., \& Kung, C. F. (1975). The singularly perturbed linear state regulator problem II. SIAM J. Control, 13, 327 - 337. http://dx.doi.org/10.1137/0313019

Roussel, M. R. (2005). Singular Perturbation Theory. Lecture Notes.

Sethi, S. P., \& Staats, P. W. (1978). Optimal Control of Some Simple Deterministic Epidemic Models. Journal of Operational Research Society, 29(2), 129 - 136. 